\title{
KIT EDUCACIONAL PARA ESTUDO DE GERAÇÃO ELÉTRICA A PARTIR
} DE ENERGIA EÓLICÁ

\author{
Antonio Luiz Pereira de Siqueira Campos \\ Doutor em Engenharia Elétrica. Professor da UFRN. Pesquisador do CNPq - Nível 2. E- \\ mail: Antonio.luiz@ pq.cnpq.br. \\ Aécio Vinícius Amorim Farias \\ Técnico em Eletrônica do IFRN. E-mail: Aécio.vinicius@ifrn.edu.br. \\ Débora Raiane Farias de Azevedo Fernandes \\ Alunos do Curso de Manutenção de Computadores do IFRN \\ Felipe José Santana de Sales \\ Alunos do Curso de Manutenção de Computadores do IFRN \\ Francelio Ferreira \\ Alunos do Curso de Manutenção de Computadores do IFRN \\ Juliana Ramos da Silva \\ Alunos do Curso de Manutenção de Computadores do IFRN \\ Manassés Albuquerque de Sousa Melo \\ Alunos do Curso de Manutenção de Computadores do IFRN
}

\begin{abstract}
RESUMO
Vários estudos desenvolvidos nos últimos anos apontam para as implicações e impactos sócio-ambientais do consumo de energia. Fontes de energias renováveis são apresentadas como alternativa para atender as demandas da sociedade com qualidade, segurança e redução dos danos ambientais decorrentes do consumo de energia. Devido ao crescimento de sua importância, este artigo apresenta uma proposta de kit educacional para ensino do uso de energia eólica na geração de energia elétrica. Inicialmente, a evolução do aproveitamento da energia eólica é descrita, sendo apresentadas algumas experiências sobre o ensino desse tipo de tecnologia nos níveis médio e superior. A proposta é apresentada, mostrando-se resultados obtidos com o aerogerador tais como tensão gerada e circuitos eletrônicos utilizados. Algumas práticas são sugeridas e, por fim, as principais conclusões acerca do sistema são apresentadas.
\end{abstract}

PALAVRAS-CHAVE: Energia Eólica, Aerogerador, Kit Educacional.

\section{EDUCATIONAL KIT FOR STUDY OF ELECTRICAL GENERATION FROM WIND POWER}

\begin{abstract}
Several studies developed in recent years point to the implications and socioenvironmental impacts of energy consumption. Renewable energy sources are presented as an alternative to meet the demands of society with quality, safety and reduce environmental damage arising from energy consumption. Due to the increase of its importance, this paper presents a proposal for educational kit for teaching the use of wind power to generate electricity. Initially, the evolution of the use of wind energy is described and some experiences on the teaching of this type of energy in high school and college are presented. The proposal is presented; showing up results with the Wind turbine such as
\end{abstract}


voltage generated and electronic circuits used. Some practices are suggested and, finally, the main conclusions about the system are presented.

KEY-WORDS: Wind Energy, Wind turbine, Educational Kit. 


\section{KIT EDUCACIONAL PARA ESTUDO DE GERAÇÃO ELÉTRICA A PARTIR DE} ENERGIA EÓLICẢ

\section{INTRODUÇÃO}

Energia eólica é a energia cinética contida nas massas de ar em movimento (vento). Seu aproveitamento ocorre através da conversão da energia cinética de translação em energia cinética de rotação, com o emprego de turbinas eólicas, também denominadas aerogeradores, para a geração de energia elétrica, ou através de cataventos e moinhos para trabalhos mecânicos, como bombeamento de água (ANEEL, 2002).

Da mesma forma que a energia hidráulica, a energia eólica é utilizada há milhares de anos, com as mesmas finalidades, a saber: bombeamento de água, moagem de grãos e outras aplicações que envolvem energia mecânica. Para a geração de eletricidade, as primeiras tentativas surgiram no final do Século XIX, mas somente um século depois, com a crise internacional do petróleo (década de 1970), é que foram despertados interesses e investimentos suficientes para viabilizar o desenvolvimento e aplicação de equipamentos em escala comercial (ANEEL, 2002).

A primeira turbina eólica comercial ligada à rede elétrica pública foi instalada em 1976, na Dinamarca. Atualmente, existem mais de 30 mil turbinas eólicas em operação no mundo. Em 1991, a Associação Européia de Energia Eólica estabeleceu como metas a instalação de 4.000 MW de energia eólica na Europa até o ano 2000 e 11.500 MW até o ano 2005. Essas e outras metas estão sendo cumpridas muito antes do esperado (4.000 MW em 1996, 11.500 MW em 2001). As metas atuais são de 40.000 MW na Europa até 2010. Nos Estados Unidos, o parque eólico existente é da ordem de $2.500 \mathrm{MW}$ e prevê-se uma instalação anual em torno de 1.500 MW para os próximos anos (ANEEL, 2002).

O custo dos equipamentos, que se caracterizava como um dos principais entraves ao aproveitamento comercial da energia eólica, foi bastante reduzido entre os anos 1980 e 1990. Estimativas conservadoras indicam que o custo de uma turbina eólica moderna está em torno de US\$1.000,00 por kW instalado. Os custos de operação e manutenção variam de US\$ 0,006 a US\$ 0,01 por kWh de energia gerada, nos dez primeiros anos, e de US\$ 0,015 a US\$ 0,02 por kWh, após dez anos de operação (ANEEL, 2002).

Recentes desenvolvimentos tecnológicos (sistemas avançados de transmissão, melhor aerodinâmica, estratégias de controle e operação das turbinas, etc.) têm reduzido custos e melhorado o desempenho e a confiabilidade dos equipamentos. Espera-se, portanto, que a energia eólica venha a ser muito mais competitiva economicamente na próxima década (ANEEL, 2002).

Este trabalho tem por objetivo apresentar um kit educacional desenvolvido na Unidade Zona Norte de Natal, do IFRN, que pode ser utilizado em aulas práticas de disciplinas que ministrem conteúdos sobre fontes alternativas de energia, física, geração de energia, etc. $\mathrm{O}$ kit foi desenvolvido a partir do projeto apresentado em (MUSSEL, 2008) e foi aprimorado para permitir a realização de várias práticas. Serão apresentados alguns detalhes do kit, bem como, algumas atividades práticas que podem ser desenvolvidas. 


\section{USO DA ENERGIA EÓLICA NO ENSINO MÉDIO E/OU SUPERIOR}

$\mathrm{Na}$ literatura, artigos que mostram diversas abordagens pedagógicas sobre a energia eólica podem ser encontrados. Em (MARTINS, GUARNIERI, e PEREIRA, 2008), os autores apresentam uma revisão dos conceitos físicos relacionados ao emprego da energia cinética dos ventos na geração de eletricidade.

Em (NASCIMENTO, 2006), o autor apresenta um relato sobre as atividades desenvolvidas por alunos de graduação no âmbito do Laboratório de Fontes Alternativas de Energia (LAFAE). O setor eólico desse laboratório vem desenvolvendo um projeto denominado "Banco de Dados de Vento junto ao Ministério de Minas e Energia". O projeto compreende a pesquisa e o tratamento de informações eólicas do território nacional. Parte desse projeto é de responsabilidade do LAFAE.

Em (WEIGMANN et al., 2006) os autores utilizaram conhecimentos adquiridos na unidade de estudos de Sistemas de Energia, do Curso Superior de Tecnologia em Sistemas Digitais do IFSC e apresentaram um estudo das fontes de energias renováveis e suas formas de geração. Os projetos de pesquisa de fontes alternativas de energias renováveis do IFSC possibilitam aos alunos uma oportunidade de conhecer e utilizar fontes de energia alternativas. Na Unidade de Florianópolis do IFSC encontra-se em fase de implantação, a instalação de duas turbinas eólicas.

A necessidade de relacionar o processo de ensino-aprendizagem, em física, visando formar uma cultura científica e ambiental levou (JAPIASSÚ JUNIOR, 2005) a escolher temas do currículo da física do ensino médio, pertinentes às tecnologias de fontes alternativas de energia, para elaboração de roteiros de aulas experimentais permitindo ao aluno reconhecer o papel da física no sistema produtivo. Neste trabalho, o autor descreveu uma possível metodologia a ser usada em aulas práticas, na qual o trabalho é dividido em quatro etapas. $\mathrm{Na}$ primeira é feito um minucioso levantamento bibliográfico. $\mathrm{Na}$ segunda etapa são elaborados roteiros de aulas práticas, nos quais são descritos experimentos. A terceira etapa consiste na operacionalização de aulas. A quarta etapa é a obtenção de dados.

Em (SOUZA, 2006) os autores objetivaram estudar o aproveitamento da energia eólica e relacionar os princípios físicos envolvidos no funcionamento de uma turbina eólica (catavento) no ensino de física, e se possível à montagem de uma mini-estação de produção de energia eólica.

Em (SOUSA, 2005), o autor levanta que, devido à sua abrangência e relevância para o ensino das ciências, o conceito de energia deve ser apresentado desde cedo na escola. Por isso, ele desenvolveu um trabalho que teve como finalidade fornecer material didático em forma de info-gráficos, para que professores possam trabalhar o ensino de energia qualitativamente.

\section{DESCRIÇÃO DO KIT EDUCACIONAL}

Os trabalhos encontrados na literatura e as dificuldades levantadas no desenvolvimento de práticas com fontes alternativas de energia levaram ao desenvolvimento de um kit educacional para energia eólica. O kit poderá ser usado em diversas disciplinas lecionadas no IFRN, como: Física, Eletrotécnica, etc. 
O kit educacional desenvolvido foi baseado na turbina eólica apresentada em (MUSSEL, 2008). Diversas modificações e melhorias foram feitas. A turbina construída baseia-se no modelo desenvolvido pelo engenheiro finlandês Savonius em 1922. Sua idéia era de montar dois semi-cilíndros sobre um eixo vertical. A turbina é de fácil construção e pode aceitar vento de qualquer direção. No entanto, essa turbina foi ligeiramente menos eficiente que as turbinas mais comuns de eixo horizontal (MUSSEL, 2008). A Figura 1 ilustra o kit montado, indicando suas partes componentes. Ele é composto de um ventilador cuja velocidade pode ser controlada, um gerador de indução, a turbina eólica e protoboard com componentes eletrônicos, para práticas de retificação e filtragem.

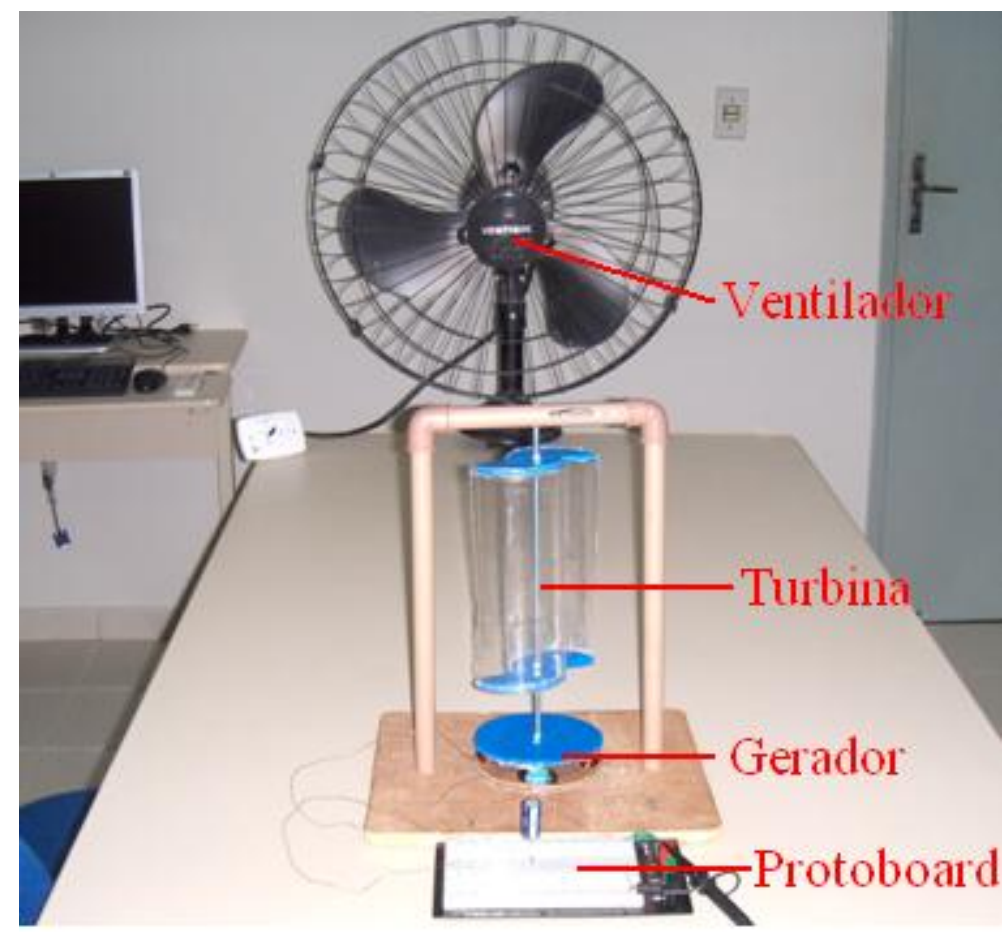

Figura 1 - Visão geral do kit educacional.

Esse modelo de turbina eólica gera eletricidade com um simples gerador que produz uma corrente pulsada. Isso é possível devido à indução magnética, pois cada vez que um ímã passa sobre uma bobina, tem-se uma tensão induzida nos terminais desta. Com quatro bobinas ligadas entre si em série, o resultado é que a tensão induzida pode ser quatro vezes maior. Esta é, possivelmente, a forma mais simples e eficiente para gerar eletricidade e é o mesmo princípio básico utilizado em quase todas as turbinas eólicas.

Para controlar a velocidade do ventilador foi utilizado um dimmer comercial, que permite que a velocidade varie de 0 até a máxima fornecida pelo ventilador. A Figura 2 ilustra o dimmer usado, ele permite a variação da velocidade do ventilador de forma contínua.

A Figura 3 ilustra a máquina de indução. Foram utilizados quatro imãs de hard disk (HD) e foram fabricados quatro enrolamentos. Optou-se por esse arranjo devido sua simplicidade. Pela Figura 3, pode-se observar que o disco dos imãs foi fabricado com material de pastas escolares (Figura 3(a)) e o disco dos enrolamentos foi feito com papelão (Figura 3(b)). 


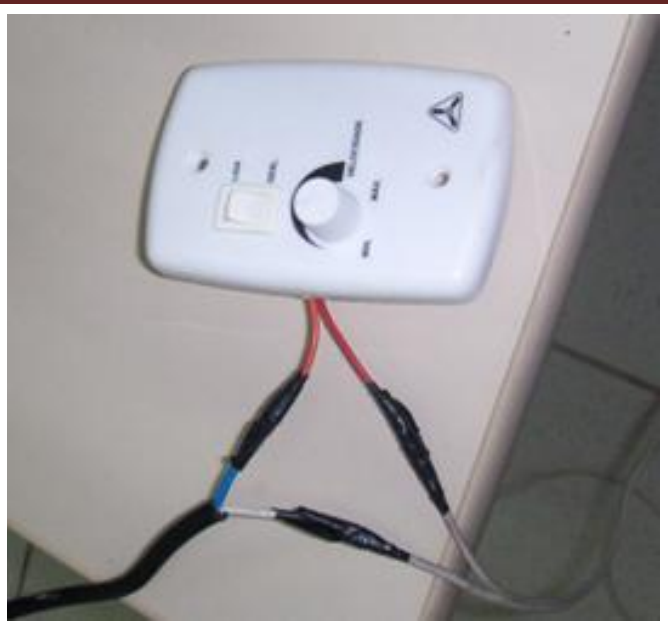

Figura 2 - Dimmer para controlar a velocidade do ventilador.

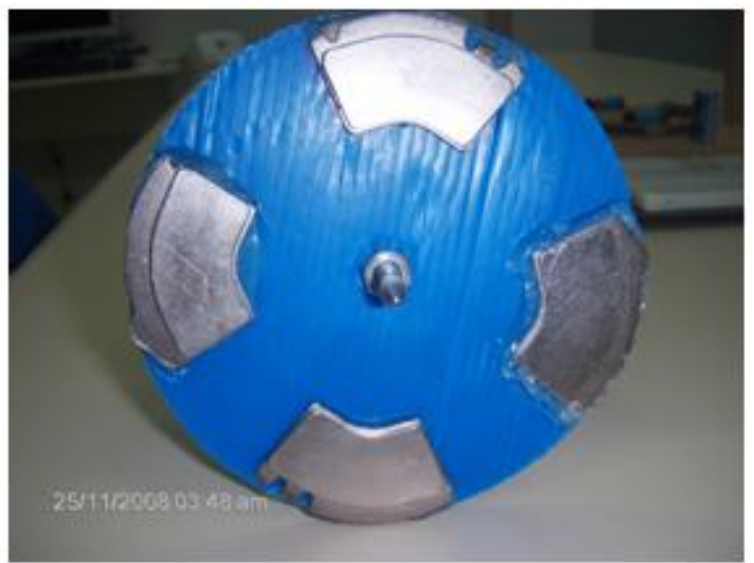

(a)

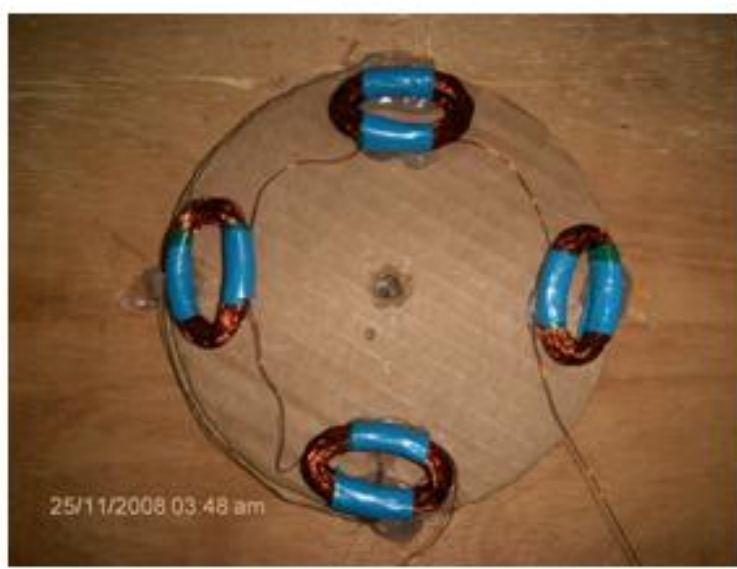

(b)

Figura 3 - Gerador de indução: (a) Disco com imãs de HD e (b) Disco com enrolamentos.

\section{PRÁTICAS REALIZADAS}

O kit educacional permite que diversas práticas sejam realizadas. A seguir, são apresentadas algumas delas. 


\section{a) Influência da velocidade do vento}

A quantidade de energia transferida para uma turbina eólica é diretamente proporcional à área varrida pelo rotor, à densidade do ar e ao cubo da velocidade do vento. A potência $\mathrm{P}$ do vento pode ser dada por (WIKIPEDIA, 2008):

$$
P=\frac{1}{2} \alpha \rho r^{2} v^{3}
$$

em que $P$ é a potência em watts, $\alpha$ é um fator eficiência determinado pela concepção da turbina, $\rho$ é a densidade de massa do ar em quilogramas por metro cúbico, $r$ é o raio da turbina eólica em metros, e $v$ é a velocidade do ar em metros por segundo.

Da Equação (1) pode-se ver que a tensão gerada nos terminais do estator será proporcional à:

$$
V \propto v^{3 / 2}
$$

equação (2)

Para comprovar isso, o ventilador foi ligado na metade da velocidade. Com o auxílio de um osciloscópio digital da Tektronix TDS 1002B, os parâmetros da tensão gerada foram medidos. Na Figura 4 pode-se observar esses valores. A frequência da tensão gerada foi de $14,09 \mathrm{~Hz}$ e o valor eficaz foi de $0,79 \mathrm{~V}$.

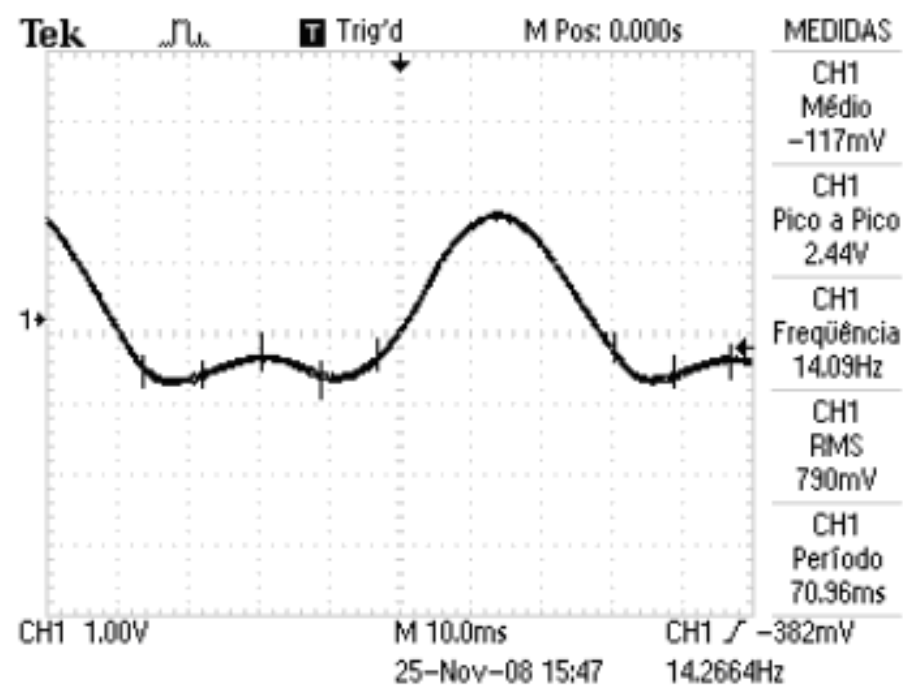

TDS 1002B - 15:46:58 25/11/2008

Figura 4 - Tensão gerada com metade da velocidade máxima do ventilador.

Aumentando-se a velocidade do ventilador para a máxima as medições feitas para a Figura 4 foram repetidas. Pode-se observar na Figura 5 os valores da tensão gerada. A frequência aumentou para $25,38 \mathrm{~Hz}$ e o valor eficaz da tensão aumentou para $1,50 \mathrm{~V}$. 


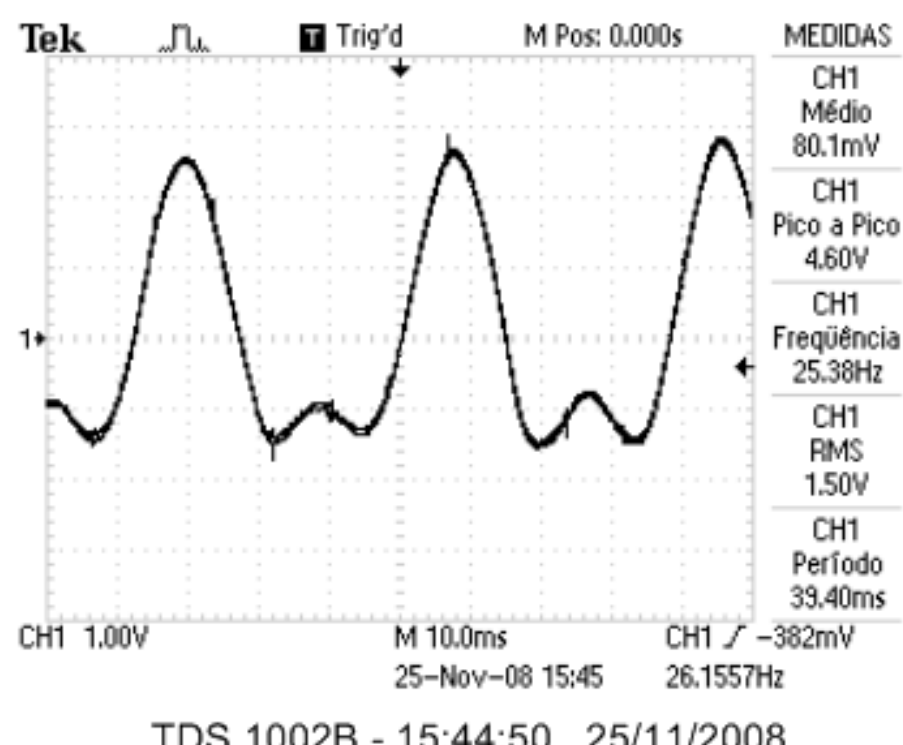

Figura 5 - Tensão gerada para a velocidade máxima do ventilador.

Pode-se observar uma relação entre as freqüências de 1,8. Considerando uma relação linear entre a freqüência elétrica, a freqüência mecânica e a velocidade do vento; pode-se aplicar esse fator na Equação (2) e para se obter um aumento na tensão gerada de 2,4. Aplicando esse fator na tensão RMS, dever-se-ia ter uma tensão gerada de 1,90V. Esse valor é próximo do valor medido na Figura 5, que foi de $1,50 \mathrm{~V}$. Vale salientar que não está sendo considerada nenhuma constante de proporcionalidade.

\section{b) Retificação de meia-onda e filtragem capacitiva}

A segunda prática realizada foi a verificação da retificação de meia-onda e a filtragem capacitiva. Foi montado o circuito mostrado na Figura 6. O circuito é composto por um diodo retificador, um resistor e um filtro capacitivo.

A tensão na saída do gerador foi ilustrada na Figura 5. Essa tensão foi aplicada na entrada do diodo. Como se pode ver, a forma de onda é alternada e possui valor médio igual a $80 \mathrm{mV}$.

Ao passar o sinal ilustrado na Figura 5 através do diodo, a onda alternada torna-se uma onda pulsante com valor médio igual a $295 \mathrm{mV}$. A tensão retificada é ilustrada na Figura 7. Esse processo aumentou em quase quatro vezes o valor médio da tensão.

Após o diodo, a tensão é aplicada a um filtro capacitivo. Esse filtro reduz a pulsação do sinal retificado, elevando bastante o valor médio, tornando o sinal próximo de um sinal de corrente contínua. $\mathrm{O}$ valor médio da tensão aumentou para $1,64 \mathrm{~V}$, como pode-se observar na Figura 8.. 


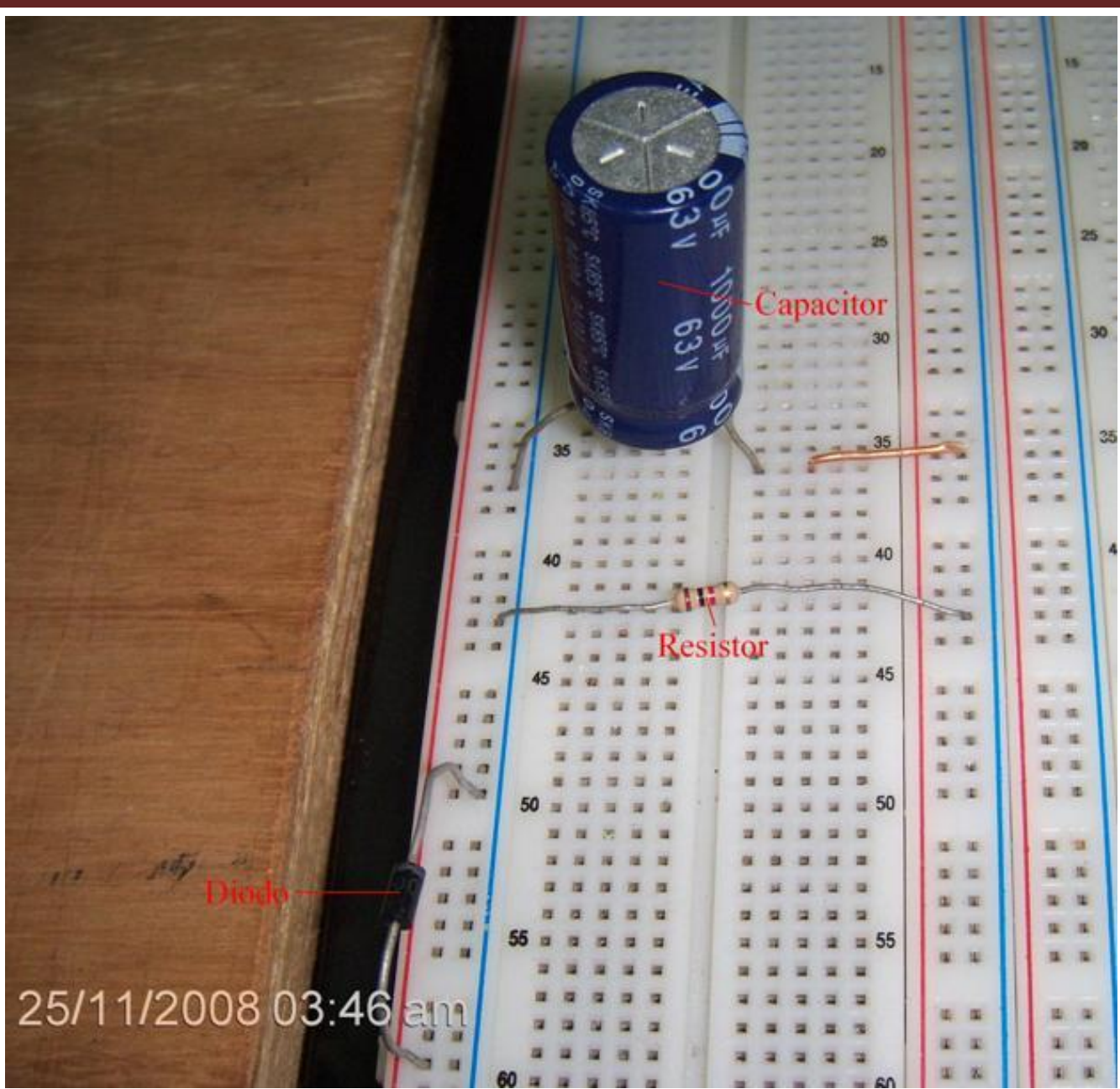

Figura 6 - Circuito retificador montado.

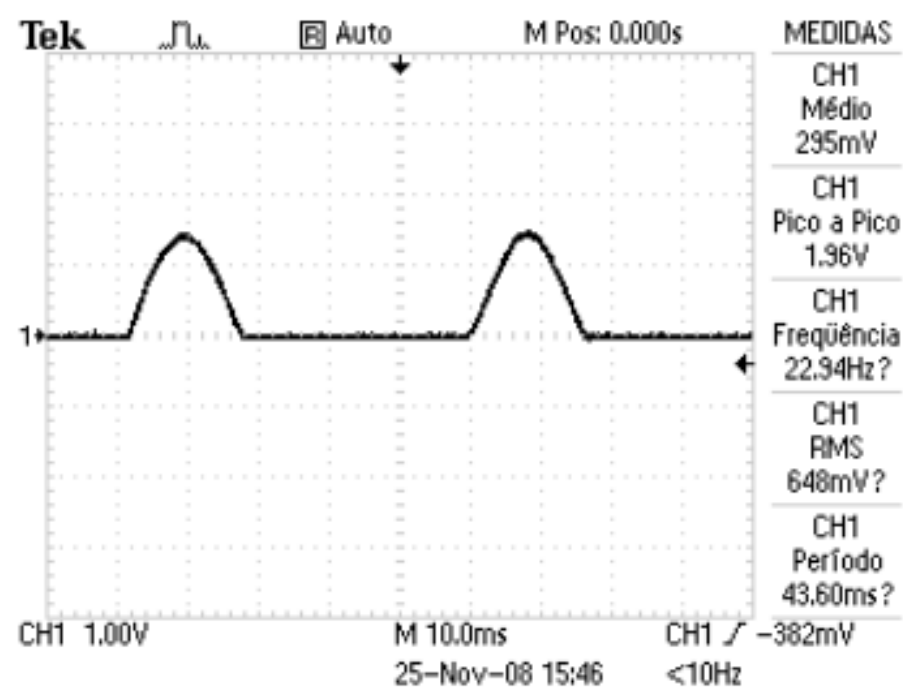

TDS 1002B - 15:45:40 25/11/2008

Figura 7 - Tensão retificada. 


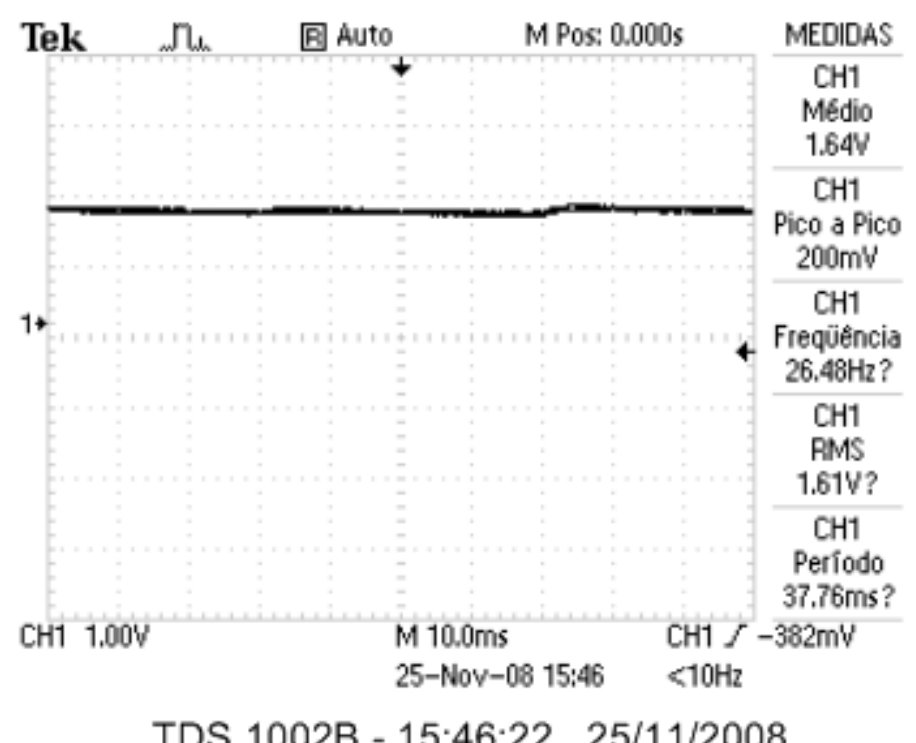

Figura 8 - Tensão filtrada.

\section{CONCLUSÕES}

Neste artigo foi apresentado um kit educacional de baixo custo para se estudar a geração de energia elétrica a partir de energia eólica. O kit educacional foi desenvolvido no Campus Natal - Zona Norte, do IFRN. O kit permite que diversas práticas sejam realizadas. Duas possíveis práticas foram apresentadas nesse artigo. Práticas sobre indução magnética e lei de Lenz também podem ser realizadas. Pretende-se tornar o kit mais industrial, inserindo materiais melhores e mais práticas. Outros tipos de hélices, como as utilizadas em turbinas de eixo horizontal, também podem ser exploradas.

\section{REFERÊNCIAS}

1. ANEEL, Atlas de energia elétrica do Brasil. Agência Nacional de Energia Elétrica, Brasília, 2002.

2. JAPIASSÚ JUNIOR, F., Energia Solar, Eólica e Biomassa no Ensino de Física. Anais do XVI Simpósio Nacional de Ensino de Física. Rio de Janeiro, Janeiro, 2005.

3. MARTINS, F. R., GUARNIERI, R. A. e PEREIRA, E.B., O Aproveitamento da Energia Eólica. Revista Brasileira de Ensino de Física, v.30, n.1, pp. 1 - 13, 2008.

4. MUSSEll, D., Build Your Own Wind Turbine. Disponível em: www.reenergy.ca. Acessado em outubro de 2008.

5. NASCIMENTO, J. L., A Base de Informações sobre Energia: Um Projeto de Atividades Integradoras do Laboratório de Fontes Alternativas de Energia LAFAE. Anais do XXXIV Congresso Brasileiro de Ensino de Engenharia. Passo Fundo, Setembro de 2006.

6. SOUZA, E. S., SOUSA, J. J. F. e BARROS, S. S., Material Didático Para O Ensino Do Conceito De Energia Na Aula De Ciências Da Escola Fundamental, Anais do XVI Simpósio Nacional de Ensino de Física. Rio de Janeiro, Janeiro, 2005. 
7. SOUZA, W. G.. Energia eólica e o Ensino de Física. Trabalho de Conclusão de Curso. 2006. Centro Universitário de Caratinga. Caratinga - MG.

8. WEIGMANN, P. R., NAVARRO, A. E., CEZÁRIO, I. C., PAZETO, L. W., SILVA, R. L. e STEINBACH, R., As Fontes Alternativas de Energia no CEFET/SC. Revista Principia, João Pessoa, n.13, pp. 76 - 81. Abril, 2006.

9. WIKIPEDIA, Wind Turbine. Disponível em: http://en.wikipedia.org/wiki/Wind_turbine. Acessado em 25/11/2008. 